ISSN 0103-9954

\title{
SIMULAÇÃO E AVALIAÇÃO ECONÔMICA DE REGIMES DE DESBASTES E DESRAMA PARA OBTER MADEIRA DE Pinus taeda LIVRE DE NÓS
}

\section{SIMULATION AND ECONOMIC EVALUATION OF THINNING REGIMES AND PRUNING TO OBTAIN Pinus taeda WOOD FREE FROM KNOTS}

\author{
José Roberto S. Scolforo ${ }^{1}$ Fausto Weimar Acerbi Júnior ${ }^{2}$ \\ Antônio Donizette de Oliveira ${ }^{3}$ Romualdo Maestri ${ }^{4}$
}

\begin{abstract}
RESUMO
Este estudo teve como objetivo simular e avaliar economicamente regimes de desbastes e de desrama para Pinus taeda, para obtenção de madeira livre de nós (clearwood) e de madeira para múltiplos usos. A área em estudo está situada no município de Jaguariaíva - PR. Foram testados dois cenários com diferentes números, épocas e intensidade de desbaste, com base em diferentes densidades iniciais de plantio, em diferentes níveis de produtividade, almejando a produção de madeira desramada, livre de nós, e de madeira não-desramada para vários usos. Os regimes de manejo gerados foram submetidos à análise de investimento a três taxas de desconto e a seis diferentes opções de preço da madeira desramada em relação à madeira não desramada, o que totalizou 86.022 diferentes opções de manejo. As simulações de crescimento, produção e desbastes foram obtidas com base no software PISAPRO. As análises econômicas foram realizadas valendo-se da maximização do valor presente líquido para uma série de infinitas rotações, utilizando, para tal, o software INVEST. Os regimes de manejo que apresentaram maiores rentabilidades, para as classes de sítio I e II e para os custos de produção e preços estipulados, foram aqueles com plantio de 833 árvores/ha e com corte final aos 21 anos, após a realização de três desbastes: sendo o primeiro um pré-comercial seguido de desrama aos 4 anos, mantendo 500 árvores/ha, o segundo um comercial aos 12 anos, mantendo 400 árvores/ha e o terceiro um comercial aos 14 anos mantendo 100 árvores/ha. Para a classe de sítio III, a melhor opção foi a adoção de regimes de manejo com plantio de 1.111 árvores/ha e corte final aos 20 anos, após a realização de um desbaste pré-comercial seguido de desrama aos 4 anos, mantendo 500 árvores/ha e de um desbaste comercial aos 12 anos, mantendo 200 árvores/ha. A segunda desrama, para as três classes de sítio, foi realizada entre 7 e 8 anos de idade.
\end{abstract}

Palavras-chave: simulação, desbastes, desrama, análise de investimento.

1. Engenheiro Florestal, Dr., Professor Titular do Departamento de Ciências Florestais, Universidade Federal de Lavras, Caixa Postal 37, CEP 37200-000, Lavras (MG).

2. Engenheiro Florestal, MSc., Professor Assistente do Departamento de Ciências Florestais, Universidade Federal de Lavras, Caixa Postal 37, CEP 37200-000, Lavras (MG).

3. Engenheiro Florestal, Dr., Professor Adjunto do Departamento de Ciências Florestais, Universidade Federal de Lavras, Caixa Postal 37, CEP 37200-000, Lavras (MG).

4. Engenheiro Florestal, Gerente Técnico da Empresa Pisa Florestal S.A., Rodovia PR 151, Km 232, CEP 84200-000, Jaguariaíva (PR). 


\begin{abstract}
This study had the objective of simulating and evaluating economically thinning regimes and pruning for Pinus taeda, to obtain clearwood and wood for multiple uses. The area of study, is located in the county of Jaguariaiva, State of Paraná - Brazil. Two scenarios were tested with different numbers, time and thinning intensities from different initial planting densities, at different levels of productivity, aiming at the production of pruning wood, clearwood and no pruned wood for several uses. The generated management regimes were submitted, during the investment analysis, to 3 discount rates and for 6 different options of pruned wood prices related to no pruned wood, totaling 86,022 different management options. The growth, yield and thinning simulations were made by the PISAPRO software. The economic analyses were performed through the maximization of the present net value by using the INVEST software. The management regimes, which presented highest profitability for class I and II sites and for the stipulated prices, were those with 833 trees per hectare initially, and with final felling at 21 years after 3 thinning operations: a pre-commercial thinning followed by pruning at the age of 4 maintaining 500 trees/ha, a commercial thinning at the age of 12 maintaining 400 trees/ha and a third thinning (commercial) at the age of 14 maintaining 100 trees/ha. For the site III, the best option was the adoption of management the age of regimes with 1111 planted trees/ha and final cutting at 20 years, after the accomplishment of a pre-commercial thinning followed by pruning at the age of 4 maintaining 500 trees/ha and a commercial thinning at the age of 12 maintaining 200 trees/ha. The second pruning for the 3 classes for the site III was achieved between 7 and 8 years of age.
\end{abstract}

Key words: simulation, thinning, pruning, investment analysis.

\title{
INTRODUÇÃO
}

A perspectiva de estabilização e globalização da economia, aliada às previsões de falta de madeira de qualidade e com grandes dimensões no mercado internacional, têm levado os empresários do setor florestal a reverem seus conceitos estratégicos, buscando reduzir custos e, principalmente, agregar valor ao produto final, tornando suas empresas cada vez mais competitivas para, assim, poderem aproveitar as oportunidades presentes e futuras no mercado de produtos florestais.

Um exemplo dessa estratégia é observado em países como o Japão, EUA, Canadá e Suécia onde ocorrem os mais altos índices de transformação de madeira bruta em produtos mais valorizados (MACEDO \& MATTOS, 1995).

Seguindo a tendência mundial, instalaram-se nos últimos anos, no sul do Brasil, algumas indústrias, objetivando a produção de madeira serrada de Pinus livre de nós. Essas indústrias, no entanto, aproveitam o mesmo tipo de toras que as demais, por escassez de madeira desramada no mercado, e no seu processo industrial produzem painéis colados, "clear blocks", "finger joint" etc., retirando os nós na sua manufatura e conseguindo, com isso, alto valor para tais produtos (SCOLFORO \& MAESTRI, 1997).

Segundo BERTOLOTI et al.. (1983), um dos maiores problemas, encontrados na produção 
de madeira para serraria nos EUA, é a ocorrência de defeitos internos no fuste, não sendo possível detectá-los na árvore em pé. Como consequiência, grande soma de recursos é aplicada na exploração, transporte, secagem e desdobro da madeira de baixa qualidade. Torna-se imprescindível, portanto, a aplicação correta de diferentes técnicas de desbaste e desrama, visando à obtenção de madeira com boa qualidade para serraria, laminação ou faqueado.

Existem diferentes alternativas para viabilizar estudos sobre a melhor estratégia de desbaste. Uma delas foi apresentada por SCHNEIDER (1984 e 1993) em que a tônica é determinar um fator K e uma Constante Regulativa C, propostos por MAGIN (1964), para definir o tipo e a intensidade dos desbastes a serem realizados no povoamento. SCHNEIDER (1993), utilizando o fator K-Magin 0,74 e a Constante Regulativa C 8,2 para desbaste moderado a forte e o fator K-Magin 0,82 e a Constante Regulativa C 8,8 para desbaste forte a extremamente forte, encontrou erros médios na estimativa do volume desbastado variando de $3,7 \%$ a $5,8 \%$ e de $-0,2 \%$ a $3,7 \%$ para desbaste moderado a forte e desbaste forte a extremamente forte respectivamente.

Seguindo essa linha conceitual FINGER e SCHNEIDER (1999) estudaram o comportamento do Índice de Espaçamento Relativo ( $\mathrm{S} \%$ ) e verificaram a sua eficiência como indicador do peso do desbaste a ser realizado em uma floresta de Eucalyptus grandis, uma vez que os autores detectaram que a magnitude deste Índice diminui para uma mesma altura dominante com o aumento da área basal mantida após o desbaste, possibilitando descrever o Índice de Espaçamento Relativo para diferentes pesos de desbaste e altura dominante de acordo com o desenvolvimento do povoamento.

Outra alternativa é como a apresentada por BURKHART et al. (1981), BAILEY \& WARE (1983), HAFLEY \& BUFORD (1985), KNOEBELL et al. (1986), BURKHART (1987), SCOLFORO (1990), OLIVEIRA (1995), SCOLFORO \& MACHADO (1996) e ACERBI JUNIOR (1998) em que se procura modelar a estrutura da floresta por meio de uma função de distribuição com um algoritmo de desbaste a ela associado, o que possibilita testar um amplo espectro de alternativas de intensidade, época e número de desbastes, de maneira quantitativa.

A viabilidade de usar qualquer uma das alternativas é que a modelagem do fenômeno seja eficaz e produza prognoses confiáveis dos volumes dos sortimentos desejados.

Assim, este trabalho teve como objetivo definir qual o melhor regime de manejo para Pinus taeda, por sítio, para diferentes sortimentos, inclusive aqueles com madeira livre de nós (clearwood). Para isso, inúmeras alternativas de regimes de manejo (cenários) serão simuladas e, posteriormente, analisadas mediante o uso de critérios econômico-financeiros.

\section{MATERIAL E MÉTODOS}

\section{Localização e características da área}

A área em estudo, está situada no município de Jaguariaíva, estado do Paraná, entre os paralelos $24^{\circ}$ e $24^{\circ} 30^{\prime}$ de latitude sul e os meridianos $49^{\circ} 30^{\prime}$ e $50^{\circ}$ de longitude oeste de Greenwich, com altitude variando entre 700 e $1.100 \mathrm{~m}$. 


\section{Definição dos regimes de manejo ótimos economicamente}

Dois componentes são fundamentais para que esse objetivo seja alcançado. O primeiro é um sistema de prognose do crescimento e da produção, com simulador de desbastes que seja eficiente e flexível. O segundo é uma estrutura de custos e preços reais que retrate com eficiência os cenários que serão obtidos com base no simulador de produção.

\section{Modelo para prognose do crescimento e da produção}

Para obtenção da prognose do crescimento e da produção do volume por classe diamétrica, foi utilizado o software PISAPRO (SCOLFORO, 1997), desenvolvido valendo-se de 432 parcelas permanentes, utilizando-se, para tal, a distribuição Weibull com ajuste pelo método dos momentos. Os testes, para definição deste modelo biométrico, são apresentados em SCOLFORO et al. (1999).

A seguir, é apresentado o desenvolvimento para se alcançar a compatibilidade entre a área basal estimada para o povoamento e pela distribuição Weibull cuja função densidade de probabilidade (fdp) é :

$$
f(x)=\left(\frac{c}{b}\right) \cdot\left(\frac{x-a}{b}\right)^{c-1} \cdot \exp \left[-\left(\frac{x-a}{b}\right)^{c}\right]
$$

Em que $\mathrm{a} \leq \mathrm{x}<\infty, \mathrm{a} \geq 0, \mathrm{~b}>0$ e $\mathrm{c}>0$, sendo:

$\mathrm{a}=$ parâmetro de locação;

$\mathrm{b}=$ parâmetro de escala;

$\mathrm{c}=$ parâmetro de forma;

$\mathrm{x}=$ variável de interesse, no caso diâmetro.

O método de ajuste selecionado foi o dos momentos que propiciam obter estimativas compatíveis entre o modelo do povoamento e o modelo por classe diamétrica por meio da função geral de produção por classe de diâmetro:

$$
Y_{i}=N \int_{1}^{u} g_{i}(x) f(x, \theta) \delta x
$$

\section{Em que:}

$\mathrm{Y}_{\mathrm{i}}=$ valor total por unidade de área do atributo do povoamento definido por $\mathrm{g}_{\mathrm{i}}(\mathrm{x})$;

$\mathrm{g}_{\mathrm{i}}(\mathrm{x})=$ atributo do povoamento como função de $\mathrm{x}$

$\mathrm{f}(\mathrm{x}, \theta)=$ função de densidade de probabilidade para $\mathrm{x}$

$\mathrm{N}=$ número de árvores por unidade de área

$\mathrm{l}, \mathrm{u}=$ limite inferior e superior, respectivamente, para o produto descrito por $\mathrm{g}_{\mathrm{i}}(\mathrm{x})$.

A integral apresentada, se implementada sobre a faixa de diâmetros $\mathrm{x}$, para qualquer $\mathrm{g}_{\mathrm{i}}(\mathrm{x})$, dá o valor total por unidade de área do atributo do povoamento, definido por $\mathrm{g}_{\mathrm{i}}$ (x). São exemplos de atributos do povoamento a média dos diâmetros, a área basal e o volume por unidade de área.

Estabelecendo que $\mathrm{g}_{\mathrm{i}}(\mathrm{x})$ é igual $\mathrm{a} \mathrm{x}^{\mathrm{i}}$, a obtenção do primeiro momento não-central de $\mathrm{x}$ é 
obtida como:

$$
E\left(x_{i}\right)=\int_{-\infty}^{\infty} x^{i} f(x, \theta) \delta x
$$

No caso da distribuição de diâmetro de uma população florestal, o primeiro momento não- central $\mathrm{E}(\mathrm{x})$ é estimado por $(\Sigma \mathrm{x} / \mathrm{N})=\overline{\mathrm{x}}$, ou seja, a média aritmética dos diâmetros. Já o segundo momento não-central $\mathrm{E}\left(\mathrm{x}^{2}\right)$ é estimado por $\left(\Sigma \mathrm{x}^{2} / \mathrm{N}\right)=\overline{\mathrm{x}}^{2}$. Esse valor é o diâmetro médio quadrático. Assim, os dois primeiros momentos têm interpretação em nível do povoamento e são comuns na área florestal.

O sistema de equações utilizado contempla a função Weibull, com o parâmetro "a" sendo estimado independentemente, vinculado ao diâmetro mínimo, sendo este expresso pelo modelo:

$\ln \left(D_{\min }\right)=\beta_{\mathrm{o}}+\beta_{1}(\mathrm{Dg})^{0,5}+\beta_{2} / \mathrm{N}^{0,5}+\beta_{3}(\mathrm{I} . \mathrm{Hd})^{-1}+\ln$ ei

As estimativas dos parâmetros b e c da fdp Weibull são dependentes do diâmetro médio quadrático (dg) e da média aritmética dos diâmetros ( $\overline{\mathrm{d}}$ ). Essas variáveis são obtidas como:

- Diâmetro médio quadrático $(\mathrm{dg})$

$$
\mathrm{dg}^{2}=\frac{\mathrm{G}_{2}}{0,0000785398163 \cdot \mathrm{N}_{2}}
$$

- Média aritmética dos diâmetros ( $\overline{\mathrm{d}})$

Nesse caso como, a média aritmética $(\overline{\mathrm{d}})$ e o diâmetro médio quadrático (dg) são obtidos de forma independente com base em equações ou de estimativas, é feita a amarração do cálculo da média, à variância para impedir que $\overline{\mathrm{d}}$ seja maior ou igual a dg. É então ajustado um modelo de variância dos diâmetros para, então, obter-se a média $(\overline{\mathrm{d}})$.

$$
\begin{aligned}
& \ln \left(\mathrm{dg}^{2}-\overline{\mathrm{d}}^{2}\right)=\ln \mathrm{S}^{2} \mathrm{~d}=\beta \mathrm{o}+\beta_{1} \ln \mathrm{G}+\beta_{2} \ln \mathrm{Hd}+\beta_{3}(\mathrm{I} . \mathrm{N}) / 1000 \\
& \overline{\mathrm{d}}=\left\{\left[\frac{\mathrm{G}_{2}}{0,0000785398163 . \mathrm{N}_{2}}\right]-\exp \left[\left(\ln \mathrm{S}^{2} \mathrm{~d}\right)\right]\right\}^{(1 / 2)}
\end{aligned}
$$

Em que a área basal $\left(\mathrm{G}_{2}\right)$, o número de árvores $\left(\mathrm{N}_{2}\right)$, podem ser estimados ou prognosticados por meio de modelos similares ao (3) e (4).

$$
\ln \left(G_{2}\right)=\left(\frac{I_{1}}{I_{2}}\right) \ln G_{1}+\left(\frac{\beta_{4}}{\beta_{3}}\right)\left(1-\frac{I_{1}}{I_{2}}\right)+\left(\frac{\beta_{5}}{\beta_{3}}\right) S\left(1-\frac{I_{1}}{I_{2}}\right)
$$


$\left.\mathrm{N}_{2}=\mathrm{N}_{1}\left(\mathrm{I}_{2} / \mathrm{I}_{1}\right) \beta_{1} \cdot \exp \left[\beta_{\mathrm{o}}+\beta_{2} \mathrm{~S}\right)\left(\mathrm{I}_{2}-\mathrm{I}_{1}\right)\right]$

As duas equações para os dois parâmetros do sistema são:

$$
\begin{aligned}
& \overline{\mathrm{d}}=\int_{0}^{\infty} \mathrm{xf}(\mathrm{x}, \mathrm{b}, \mathrm{c}) \delta \mathrm{x}=\mathrm{b} \Gamma[1+(1 / \mathrm{c})] \Rightarrow \mathrm{b}=\overline{\mathrm{d}} / \Gamma(1+1 / \mathrm{c}) \\
& \mathrm{G}_{2}=0,0000785398163 . \mathrm{N}_{2} \int_{0}^{\infty} \mathrm{x}^{2} \mathrm{f}(\mathrm{x}, \mathrm{b}, \mathrm{c}) \delta \mathrm{x}=\mathrm{b}^{2} \Gamma[1+(2 / \mathrm{c})]
\end{aligned}
$$

A variância e o coeficiente de variação são dados respectivamente por:

$$
\begin{aligned}
& \mathrm{Sd}^{2}=\mathrm{dg}^{2}-\overline{\mathrm{d}}^{2}=\mathrm{b}^{2}\left[\Gamma(1+2 / \mathrm{c})-\Gamma^{2}(1+1 / \mathrm{c})\right] \\
& \mathrm{CV}=\frac{\mathrm{Sd}}{\overline{\mathrm{d}}}=\frac{\left[\Gamma(1+2 / \mathrm{c})-\Gamma^{2}(1+1 / \mathrm{c})\right]^{1 / 2}}{\Gamma(1+1 / \mathrm{c})}
\end{aligned}
$$

Obtidas as estimativas da média aritmética dos diâmetros e do diâmetro médio quadrático, o coeficiente de variação é função somente de "c" e pode ser obtido por simples técnica iterativa. Com "c" conhecido, "b" pode ser estimado como:

$$
\mathrm{b}=\frac{\overline{\mathrm{d}}}{\Gamma\left(1+\frac{1}{\mathrm{c}}\right)}
$$

O algoritmo de desbaste foi adaptado por KNOEBELL et al. (1986), SCOLFORO (1990) e SCOLFORO (1998a). Esses autores, utilizando a distribuição Weibull numa amostra que sofreu desbaste, verificaram que, embora as distribuições preditas se aproximassem das distribuições observadas, algumas discrepâncias foram verificadas entre a tabela do povoamento desbastado e a tabela do povoamento não-desbastado. Por exemplo:

a) O número de árvores preditas aumenta em algumas classes de diâmetro após o desbaste.

b) Em algumas circunstâncias a tabela do povoamento desbastado tem um diâmetro máximo maior que a do povoamento sem-desbaste, ou mesmo um menor diâmetro mínimo que a do povoamento sem-desbaste.

Portanto ficou evidenciado que as predições da distribuição de diâmetro antes e após o desbaste por baixo não podem ser executadas independentemente, mas, sim, condicionadas a algum algoritmo de desbaste para que as inconsistências mencionadas não mais ocorram.

A alternativa obtida foi que a distribuição diamétrica seja efetuada antes do desbaste e, então, a proporção de área basal, em cada classe de diâmetro, seja removida para simular o desbaste. Com esse procedimento é impossível o número de árvores aumentar numa determinada classe de diâmetro após efetuar-se o desbaste. Conseqüentemente, o diâmetro mínimo pode somente aumentar e o diâmetro máximo pode somente diminuir se, em todas as classes de diâmetros, ocorrer a remoção de 
área basal.

O modelo foi definido especificando a quantidade de área basal a ser removida em cada classe de diâmetro e tem a forma:

$$
\mathrm{P}_{\mathrm{i}}=\exp \left[\mathrm{b}_{1}\left(\frac{\mathrm{d}_{\mathrm{i}}^{2}}{\mathrm{dg}^{2}}\right)^{\mathrm{b}_{2}}\right]
$$

em que:

$\mathrm{P}_{\mathrm{i}}=$ proporção de área basal removida na classe de diâmetro i

$\mathrm{d}_{\mathrm{i}}=$ centro da classe de diâmetro $\mathrm{i}$

$\mathrm{dg}=$ diâmetro médio quadrático do povoamento

$\mathrm{b}_{1}, \mathrm{~b}_{2}=$ coeficientes estimados com base nos dados

Como é considerado o desbaste por baixo, a função removerá mais árvores nas menores classes de diâmetro do que nas maiores. Assim, esse modelo, quando ajustado, representa o padrão médio de remoção de cada uma das inúmeras alternativas de desbastes já praticadas na região em estudo. No caso desse sistema, foi modelada 1 situação, incluindo desbaste pré - comercial, 14 alternativas de desbaste seletivo + sistemático na $6^{\mathrm{a}}$ linha, 14 alternativas de $1^{\mathrm{o}}$ desbaste sistemático na $10^{\text {a }}$ linha + seletivo, 15 alternativas de 2,13 de 3 e 3 de 4 desbastes, praticados em diferentes idades e com diferentes intensidades de remoção de área basal.

Uma síntese de como efetuar a prognose é apresentada a seguir:

- Predição da distribuição de diâmetros, antes do desbaste provenientes da distribuição Weibull.

- Partindo das menores classes de diâmetro remover a proporção de área basal especificada pela função de remoção.

- Caminha-se por meio das classes de diâmetro até que a área basal a ser removida seja obtida.

- Se a desejada remoção de área basal não é obtida após se caminhar por todas as classes de diâmetro, deve-se retornar às menores classes e remover a área basal remanescente naquelas classes. Procede-se, dessa maneira, por meio das classes seguintes até que o desejado nível de remoção da área basal seja obtido.

A consistência do modelo foi realizada numa grande base de dados composta de 717 estratos da própria Pisa Florestal. A diferença detectada nas estimativas, em relação ao real, foi de $4 \%$ no volume total e de 8 a $18 \%$ nos sortimentos o que assegura o uso do sistema.

\section{Alternativas de Manejo}

Foram simuladas diferentes alternativas de regimes de manejo, enquadradas em dois cenários com diferentes números, épocas e intensidade de desbaste, com base em diferentes densidades iniciais de plantio, em diferentes níveis de produtividade. Para a obtenção de madeira livre de nós, o 
primeiro desbaste foi um pré-comercial, realizado em idades jovens seguido de desrama.

A primeira desrama ocorreu simultaneamente ao desbaste pré-comercial, e as árvores selecionadas foram desramadas até uma altura mínima de $2,4 \mathrm{~m}$ desde que não ultrapasse $50 \%$ da altura da árvore ou 1/3 de sua copa viva. A segunda desrama ocorreu, normalmente, em torno dos 7 e 8 anos, atingindo uma altura de 6,7 a 7,0 m nas árvores selecionadas.

Foram então testadas opções de regimes de manejo com base em três diferentes densidades iniciais de plantio em diferentes sítios, conforme pode ser visto na Tabela 1.

TABELA 1: Diferentes densidades iniciais de plantio em diferentes sítios.

\begin{tabular}{|c|c|c|}
\hline SÍTIO & $\begin{array}{l}\text { ESPAÇAMENTO } \\
(\mathrm{m} \times \mathrm{m})\end{array}$ & $\begin{array}{l}\text { DENSIDADE INICIAL } \\
\text { DE PLANTIO }\end{array}$ \\
\hline $\mathrm{I}$ & $3 \times 2,5$ & 1.333 árvores/ha \\
\hline \multirow[t]{2}{*}{$(\mathrm{S}=28,5 \mathrm{~m})$} & $3 \times 3,0$ & 1.111 árvores/ha \\
\hline & $3 \times 4,0$ & 833 árvores/ha \\
\hline II & $3 \times 2,5$ & 1.333 árvores/ha \\
\hline \multirow[t]{2}{*}{$(\mathrm{S}=25,5 \mathrm{~m})$} & $3 \times 3,0$ & 1.111 árvores/ha \\
\hline & $3 \times 4,0$ & 833 árvores/ha \\
\hline III & $3 \times 2,5$ & 1.333 árvores/ha \\
\hline \multirow[t]{2}{*}{$(\mathrm{S}=22,5 \mathrm{~m})$} & $3 \times 3,0$ & 1.111 árvores/ha \\
\hline & $3 \times 4,0$ & 833 árvores/ha \\
\hline
\end{tabular}

a) Cenários propriamente ditos: Foram estabelecidos dois cenários com base nas nove opções definidas na Tabela 1, para os quais variou-se o número, a época de ocorrência dos desbastes précomerciais e comerciais e do corte final, e a intensidade dos desbastes.

a.1) Cenário 1: Esta é a situação em que serão realizados 2 desbastes em diferentes épocas e com diferentes intensidades de remoção, além de diferentes idades de corte final. Na Tabela 2 são mostradas as opções a serem simuladas, considerando a densidade inicial de plantio de 1.333 árvores/ha, no índice de sítio $28,5 \mathrm{~m}$. Para as outras oito situações definidas na Tabela 1, a lógica é a mesma.

Considerando qualquer densidade inicial de plantio, há 243 alternativas de regime de manejo, ou seja, na idade de 4 anos em que é realizado o desbaste pré-comercial é possível deixar 500, 550 ou 600 árvores remanescentes, sendo que o desbaste comercial pode ser realizado aos 8,10 ou 12 anos, deixando 200, 250 ou 300 árvores remanescentes e executando o corte final aos 20, 21 ou 22 anos.

Fixando-se a idade do desbaste pré-comercial aos 4 anos, é possível realizar 81 diferentes alternativas de regimes de manejo. Multiplicando-se esse valor por 3, já que o desbaste pré-comercial pode ocorrer aos 4, 5 ou 6 anos, chega-se ao número de 243 possíveis combinações. Portanto, para cada uma das nove situações da Tabela 1 existem 243 possíveis combinações, resultando em um número total de 2.187 alternativas de regimes de manejo para o Cenário 1. 
TABELA 2: Idades dos desbastes e do corte final e número de árvores remanescentes considerando uma densidade inicial de 1.333 árvores/ha, no índice de sítio $28,5 \mathrm{~m}$, para dois desbastes.

\begin{tabular}{|c|c|c|c|c|c|c|c|c|}
\hline $\begin{array}{c}\text { Idades do } \\
\text { desbaste pré- } \\
\text {-comercial (anos) }\end{array}$ & \multicolumn{3}{|c|}{$\begin{array}{l}\text { Número de árvores } \\
\text { remanescentes } \\
\left(\mathrm{ha}^{-1}\right)\end{array}$} & \multirow[t]{2}{*}{$\begin{array}{c}\text { Idades do } 2^{\circ} \\
\text { desbaste } \\
\text { (anos) }\end{array}$} & \multirow{2}{*}{$\begin{array}{c}\begin{array}{c}\text { Número de } \\
\text { árvores } \\
\text { remanescentes } \\
\left(\text { ha }^{-1}\right)\end{array} \\
200\end{array}$} & \multicolumn{3}{|c|}{$\begin{array}{l}\text { Idades de } \\
\text { Corte Final } \\
\quad(\text { anos })\end{array}$} \\
\hline \multirow{9}{*}{4} & \multirow{9}{*}{500} & \multirow{9}{*}{550} & \multirow{9}{*}{600} & & & 20 & 21 & 22 \\
\hline & & & & \multirow[t]{2}{*}{8} & 250 & 20 & 21 & 22 \\
\hline & & & & & 300 & 20 & 21 & 22 \\
\hline & & & & \multirow{3}{*}{10} & 200 & 20 & 21 & 22 \\
\hline & & & & & 250 & 20 & 21 & 22 \\
\hline & & & & & 300 & 20 & 21 & 22 \\
\hline & & & & \multirow{3}{*}{12} & 200 & 20 & 21 & 22 \\
\hline & & & & & 250 & 20 & 21 & 22 \\
\hline & & & & & 300 & 20 & 21 & 22 \\
\hline
\end{tabular}

a.2) Cenário 2: Neste caso serão realizados 3 desbastes em diferentes épocas e com diferentes intensidades de remoção, além de diferentes idades de corte final. Na Tabela 3, são mostradas as opções a serem simuladas, considerando a densidade inicial de plantio de 1.333 árvores/ha, no índice de sítio 28,5 m. Para as outras oito situações definidas na Tabela 1, foi usada a mesma lógica.

TABELA 3: Idades dos desbastes e do corte final e número de árvores remanescentes considerando uma densidade inicial de 1.333 árvores/ha, no índice de sítio $28,5 \mathrm{~m}$, para três desbastes.

\begin{tabular}{|c|c|c|c|c|c|c|c|c|}
\hline $\begin{array}{c}\text { Idade do } \\
\text { desbaste pré- } \\
\text {-comercial } \\
\text { (anos) }\end{array}$ & $\begin{array}{c}\text { Número de } \\
\text { árv. remanesc. } \\
\left(\mathrm{ha}^{-1}\right)\end{array}$ & $\begin{array}{c}\text { Idades do } \\
2^{\circ} \\
\text { desbaste } \\
\text { (anos) } \\
\end{array}$ & $\begin{array}{l}\text { Número de } \\
\text { árv. remanesc. } \\
\left(\mathrm{ha}^{-1}\right)\end{array}$ & $\begin{array}{c}\text { Idades do } \\
3^{\circ} \\
\text { desbaste } \\
\text { (anos) } \\
\end{array}$ & $\begin{array}{l}\text { Núr } \\
\text { ren } \\
\end{array}$ & $\begin{array}{l}\text { o de } \\
\text { lesc. } \\
1 \text { i) }\end{array}$ & $\begin{array}{l}\mathrm{Id} a \\
\mathrm{Col} \\
(\end{array}$ & $\begin{array}{l}\text { s de } \\
\text { Final } \\
\text { s) }\end{array}$ \\
\hline \multirow{12}{*}{4} & \multirow{12}{*}{500} & \multirow{6}{*}{10} & \multirow{3}{*}{300} & 14 & 100 & 200 & 20 & 21 \\
\hline & & & & 15 & 100 & 200 & 20 & 21 \\
\hline & & & & 16 & 100 & 200 & 20 & 21 \\
\hline & & & \multirow{3}{*}{400} & 14 & 100 & 200 & 20 & 21 \\
\hline & & & & 15 & 100 & 200 & 20 & 21 \\
\hline & & & & 16 & 100 & 200 & 20 & 21 \\
\hline & & \multirow{6}{*}{12} & \multirow{3}{*}{300} & 14 & 100 & 200 & 20 & 21 \\
\hline & & & & 15 & 100 & 200 & 20 & 21 \\
\hline & & & & 16 & 100 & 200 & 20 & 21 \\
\hline & & & \multirow{3}{*}{400} & 14 & 100 & 200 & 20 & 21 \\
\hline & & & & 15 & 100 & 200 & 20 & 21 \\
\hline & & & & 16 & 100 & 200 & 20 & 21 \\
\hline
\end{tabular}


Há 288 alternativas de regime de manejo para cada densidade inicial avaliada, ou seja, na idade de 4 anos em que é realizado o desbaste pré-comercial é possível deixar 500 ou 600 árvores e realizar o $1^{\circ}$ desbaste comercial aos 10 ou 12 anos, deixando 300 ou 400 árvores, podendo-se realizar o $2^{\circ}$ desbaste comercial aos 14, 15 ou 16 anos, deixando 100 ou 200 árvores e executando o corte final aos 20 ou 21 anos.

Fixando-se a idade do desbaste pré-comercial aos 4 anos, é possível realizar 96 diferentes alternativas de regimes de manejo. Multiplicando-se esse valor por 3 , já que o desbaste pré-comercial pode ocorrer aos 4, 5 ou 6 anos, chega-se ao número de 288 possíveis combinações. Portanto, para cada uma das 9 situações da Tabela 1 existem 288 possíveis combinações, resultando em um número total de 2.592 alternativas de regimes de manejo para o Cenário 2.

c) Geração dos regimes de manejo: Mediante o sistema PISAPRO, foi possível simular todos os regimes de manejo propostos no item (a). Os sortimentos considerados são mostrados na Tabela 4.

TABELA 4: Produtos e suas especificações de acordo com cada uso da madeira.

\begin{tabular}{l|cc|c}
\hline \multirow{2}{*}{\multicolumn{1}{c}{ Produtos }} & \multicolumn{3}{c}{ Dimensão } \\
\cline { 2 - 4 } & \multicolumn{2}{|c}{ Diâmetro de uso } & Comprimento \\
\cline { 2 - 4 } & Mínimo $(\mathrm{cm})$ & Máximo $(\mathrm{cm})$ & $(\mathrm{m})$ \\
\hline Madeira para processo & 8 & 17,9 & 2,2 \\
Madeira para serraria & 18 & 24,9 & 2,2 \\
Madeira para laminação & 25 & 34,9 & 2,2 \\
Madeira especial & $\geq 35$ & & 2,2 \\
\hline
\end{tabular}

\section{Estrutura de Custos e Preços} Estão

$\mathrm{Na}$ Tabela 5, estão representados os custos que são calculados por hectare/ano.

TABELA 5: Custos de manejo em $\mathrm{R}$ / ha, na região de Jaguariaíva.

\begin{tabular}{lcc}
\hline \multicolumn{1}{c|}{ Operações } & Ano de ocorrência & Custos (R $\$ / \mathrm{ha})$ \\
\hline 1. Infra-estrutura(estradas, aceiros,...) & 0 & 42,00 \\
2. Preparo do terreno & 0 & 201,82 \\
3. Implantação de florestas & 0 & 218,04 \\
\hline Total até o plantio & & 461,86 \\
\hline 4. Tratos culturais & 1 & 119,42 \\
5. Tratos culturais & 2 & 95,15 \\
6. Tratos culturais & 3 & 38,54 \\
7. Proteção florestal & 1 & 28,24 \\
8. Proteção florestal & 2 & 28,24 \\
9. Proteção florestal & 3 & 28,24 \\
10. Administração & $1 \mathrm{a} \mathrm{n}^{*}$ & 80,08 \\
11. Overhead & $1 \mathrm{a} \mathrm{n}^{*}$ & 25,00 \\
\hline
\end{tabular}

Em que: $\mathrm{n}^{*}=$ idade do corte final 
incluídos, nessa modalidade, os custos de implantação até o plantio, os custos de tratos silviculturais e os custos de proteção florestal. Como esses dois últimos são terceirizados, eles são computados até o terceiro ano. Incide sobre os três custos anteriores, uma taxa de administração de $10 \%$. De forma complementar, durante toda a vida do povoamento, ocorre também o custo de administração direta ou overhead.

$\mathrm{Na}$ Tabela 6, são apresentados os custos de colheita (corte, arraste e carregamento) dos desbastes e do corte final que foram calculados por $\mathrm{m}^{3}$ no ano de exploração, sendo estes diferenciados de acordo com o volume médio das árvores retiradas em cada intervenção.

TABELA 6: Custos de corte, arraste e carregamento, por classe de uso e de volume, em $\mathrm{R} \$ / \mathrm{m}^{3}$ no ano de corte para a região de Jaguariaíva.

\begin{tabular}{|c|c|c|c|c|c|c|}
\hline \multirow{2}{*}{\multicolumn{2}{|c|}{$\begin{array}{c}\text { Classes de } \\
\text { Volume }\end{array}$}} & \multicolumn{5}{|c|}{ Classes de Uso } \\
\hline & & Processo $8 \vdash 18$ & Serraria $18 \vdash 25$ & Laminação 25 & $\longmapsto 35$ & Madeira Especial $\geq 35$ \\
\hline 0,10 & 0,20 & 8,523 & 8,631 & 8,708 & & 8,708 \\
\hline 0,20 & 0,33 & 7,154 & 7,261 & 7,338 & & 7,338 \\
\hline 0,33 & 0,46 & 6,923 & 7,031 & 7,108 & & 7,108 \\
\hline 0,46 & 0,59 & 7,154 & 7,261 & 7,338 & & 7,338 \\
\hline \multicolumn{2}{|c|}{$\geq 0,59$} & 7,415 & 7,523 & 7,600 & & 7,600 \\
\hline
\end{tabular}

Para o desbaste pré-comercial foram excluídos dos custos de exploração, os custos de arraste e carregamento uma vez que o material, fenotipicamente, inferior é cortado e deixado na floresta.

$\mathrm{O}$ custo da desrama variou de acordo com o número de árvores a serem podadas por hectare e também de acordo com a sua altura. O custo de desramar uma árvore logo após os desbastes comerciais em que esta atinge uma altura de 6,7 a 7,0 m, correspondente ao comprimento padrão de 3 toras, foi maior do que após os desbastes pré-comerciais em que a desrama atinge uma altura mínima de 2,4 m correspondente ao comprimento padrão de uma tora.

\section{Custo da terra}

Quando o plantio ocorreu em terras que pertenciam à própria empresa, considerou-se como custo anual da terra o custo de oportunidade, que é dado pelo valor dos juros sobre o preço da terra. Os valores de mercado das terras para os sítios I, II e III foram respectivamente R \$ 1.500,00/ha, R\$ $1.200,00 /$ ha e R $\$ 900,00 /$ ha.

\section{Preços dos produtos}

Os preços de venda, da madeira não desramada, dos diferentes produtos, para os quais obteve-se estimativa volumétrica, foram $\mathrm{R} \$ 10,32 ; \mathrm{R} \$ 17,70 ; \mathrm{R} \$ 24,60$ e $\mathrm{R} \$ 44,30$, respectivamente, para processo, serraria, laminação e madeira especial.

\section{Avaliação econômica dos regimes de manejo}

Foram utilizados dois critérios de análise econômica, considerando o Valor Presente Líquido de uma série infinita de rotações. Estes se diferenciam pela inclusão ou não do custo da terra. 
a) Valor Presente Líquido Geral (VPG):

O "VPG" considera o custo de oportunidade pelo uso da terra, ou seja, a terra pode ser mantida para o presente povoamento, convertida em outro povoamento ou vendida para outros usos. Esse critério pode ser expresso da seguinte forma:

$V P G=\frac{\sum_{n=0}^{t}\left(R_{n}-C_{n}\right) \cdot(1+t)^{t-n}-\left|L \cdot(1+i)^{t}-L\right|}{\left((1+i)^{t}-1\right)}$

onde:

$\mathrm{L}=$ custo da terra;

$\mathrm{t}=$ idade de rotação;

$\mathrm{R}_{\mathrm{n}}=$ receitas no ano $\mathrm{n}$;

$\mathrm{C}_{\mathrm{n}}=$ custos no ano $\mathrm{n}$;

$\mathrm{i}=$ taxa de juros comparativa ou taxa mínima de atratividade.

BENTLEY \& TEENGUARDEN (1965) e GOMES (1999), destacam que o VPG concede, para a terra, somente o seu preço de mercado presente e que algum excedente é tratado como uma renda capturada pelo proprietário acima do que ele desejaria para remunerar seu salário e capital.

b) Valor Esperado da Terra (VET)

A pressuposição peculiar do modelo de Renda do Solo ou Valor Esperado da Terra é que não existe acesso aos mercados de terra, isto é, a terra é um fator fixo de produção e, conseqüentemente, todos os excedentes econômicos resultam para a terra (BENTLEY \& TEENGUARDEN, 1965). Estabelecendo-se o Valor Presente Líquido Geral (VPG) igual a zero, o VET pode ser obtido como se segue:

$$
\operatorname{VET}=\frac{\sum_{\mathrm{n}=0}^{\mathrm{t}}\left(\mathrm{R}_{\mathrm{n}}-\mathrm{C}_{\mathrm{n}}\right) \cdot(1+\mathrm{i})^{\mathrm{t}-\mathrm{n}}}{\left((1+\mathrm{i})^{\mathrm{t}}-1\right)}
$$

Para calcular o VPG para um série de infinitas rotações, utilizou-se o software INVEST, desenvolvido por SCOLFORO (1998b) que permite simular variações nas taxas de desconto, nas distâncias de transporte, nos valores de custos e de preços dos produtos, na declividade da área explorada (acidentada ou plana) e também analisar regimes de manejo conduzidos em terras próprias ou em terras arrendadas.

\section{Análise de Sensibilidade}

Analisou-se a sensibilidade do VPG para um série de infinitas rotações dos diversos regimes de manejo a taxas de 6, 8 e $10 \%$ a.a. e a variação do preço de venda da madeira desramada em relação à madeira não-desramada. Foram analisados acréscimos de 0\%, 20\%, 40\%, 60\%, 80\% e $100 \%$ do preço de venda do produto desramado em relação ao produto não-desramado. 
Para se ter uma idéia da valorização das toras desramadas no mercado internacional, enquanto toras normais são vendidas à US\$ $100,00 / \mathrm{m}^{3}$, as sem-nós alcançam US\$ 220,00/m ${ }^{3}$, CIF posto na Coréia em 1996 (SCOLFORO e MAESTRI, 1997).

Essas simulações geraram 39.366 e 46.656 regimes de manejo para os cenários 1 e 2 respectivamente.

\section{RESULTADOS E DISCUSSÕES}

\section{Geração dos regimes de manejo para o cenário 1}

A Tabela 7 mostra os regimes de manejo mais rentáveis, economicamente, para o cenário 1 para as taxas de desconto de 6,8 e 10\% a.a. e para diferentes opções de preço de venda da madeira desramada em relação à madeira não-desramada. Verifica-se para a taxa de desconto de $6 \%$ a.a., que o investimento em sítios de alta produtividade $\left(\mathrm{S}^{1}=28,5 \mathrm{~m}\right)$ gera lucros que, dependendo do regime de manejo e do preço de mercado alcançado pela madeira podada, variam de $\mathrm{R} \$ 5.842,21$ a $\mathrm{R} \$$ 14.082,66 por hectare. Esse lucro é menor para o sítio II, com VPGs variando entre R \$3.339,34 e R \$ 11.412,94. Já para o sítio III, os lucros estão entre R \$ 1.372,32 e R \$ 8.477,38 por hectare. Esse fato evidencia que, preferencialmente, o manejo conjugado desbaste/desrama deve ser implementado em sítios mais produtivos uma vez que, nesses sítios, ocorre uma maior resposta em termos de crescimento em área basal das árvores remanescentes após a realização de cada desbaste.

Analisando as mudanças ocorridas nos VPGs dos regimes de manejo em conseqüência dos acréscimos percentuais no preço de venda da madeira desramada, pode-se inferir que para os casos de baixa ou lucratividade negativa é a viabilização de novos produtos, aliada a minimização de custos e ao uso cada vez maior de plantas com constituição genética superiores, o que poderá reverter a situação para lucro.

A densidade inicial de plantio influencia a produtividade e, consequientemente, a rentabilidade dos regimes simulados de manejo. Considerando acréscimos de até $40 \%$ no preço da madeira desramada, o plantio de 833 árvores/ha, espaçamento $3 \times 4 \mathrm{~m}$, foi o que proporcionou a maior rentabilidade para os sítios I e II. Já para o sítio III o plantio de 1.111 árvores/ha, espaçamento 3x3 m, foi o mais rentável.

Esse fato evidencia que, em sítios mais produtivos, existe uma tendência de que a melhor estratégia seja o plantio em espaçamentos mais amplos, permitindo a essas árvores o máximo aproveitamento dos nutrientes, água e luz que o sítio pode oferecer. Essas árvores apresentarão um ritmo de crescimento acelerado gerando, como produto final, peças de maiores dimensões e de

${ }^{1}$ Índice de sítio na idade de referência 20 anos obtido por meio da equação:

$$
\mathrm{S}=\operatorname{Exp}(5,7510386) \cdot\left(\frac{\mathrm{Hd}}{\operatorname{Exp}(5,75103856)}\right)^{\left(\frac{\mathrm{I}}{\mathrm{I}_{\text {Ref. }}}\right)^{0,23068992}}
$$


melhor qualidade. À medida que decresce a produtividade dos sítios, a tendência é de plantios em espaçamentos mais reduzidos, para que haja uma melhor ocupação destes. Embora ocorram mais custos, proporcionalmente o volume cresce numa maior intensidade, o que é traduzido no maior valor de VPG para essas situações.

TABELA 7: Regimes de manejo, economicamente, mais rentáveis para o Cenário 1 para taxas de desconto de 6,8 e $10 \%$ a.a. e para diferentes opções de preço de venda da madeira desramada.

\begin{tabular}{|c|c|c|c|c|c|c|c|c|c|c|c|c|c|c|c|}
\hline \multirow[t]{2}{*}{$\overline{\mathrm{APV}}$} & \multirow[t]{2}{*}{$\overline{\mathrm{DI}}$} & \multirow[t]{2}{*}{$S$} & \multirow[t]{2}{*}{$\mathrm{I}_{1}$} & \multirow[t]{2}{*}{ NR } & \multirow[t]{2}{*}{$\mathrm{I}_{2}$} & \multirow[t]{2}{*}{ NR } & \multirow[t]{2}{*}{$\mathrm{CF}$} & \multirow[t]{2}{*}{$\mathrm{VC} 1$} & \multirow[t]{2}{*}{ VC2 } & \multirow[t]{2}{*}{ VC3 } & \multirow[t]{2}{*}{ VC4 } & \multirow[t]{2}{*}{ VT } & \multicolumn{3}{|c|}{ VPG } \\
\hline & & & & & & & & & & & & & $6 \%$ & $8 \%$ & $10 \%$ \\
\hline \multirow{3}{*}{$0 \%$} & 833 & 28,5 & 4 & 500 & 12 & 200 & 20 & 40 & 65 & 197 & 547 & 857 & $5.842,21$ & $2.237,28$ & 289,1 \\
\hline & 833 & 25,5 & 4 & 550 & 10 & 200 & 21 & 36 & 70 & 158 & 450 & 720 & $3.339,34$ & 757,65 & $-601,52$ \\
\hline & 1.111 & 22,5 & 4 & 500 & 12 & 200 & 20 & 33 & 59 & 159 & 309 & 575 & $1.372,32$ & $-313,76$ & -1.194, \\
\hline \multirow{4}{*}{$20 \%$} & 833 & 28,5 & 4 & 500 & 12 & 200 & 20 & 40 & 65 & 197 & 547 & 857 & $7.399,56$ & $3.207,48$ & 932 \\
\hline & 833 & 25,5 & 4 & 500 & 8 & 200 & 20 & 33 & 57 & 113 & 446 & 720 & $4.827,37$ & $1.746,12$ & 92, \\
\hline & 1.111 & 22,5 & 4 & 500 & 12 & 200 & 20 & 36 & 71 & 158 & 410 & 683 & $2.638,16$ & 468,69 & $-679,70$ \\
\hline & 833 & 28,5 & 4 & 500 & 12 & 200 & 20 & 40 & 65 & 197 & 547 & 857 & 8.95 & $4.177,69$ & $1.575,65$ \\
\hline \multirow[t]{3}{*}{$40 \%$} & 833 & 25,5 & 4 & 500 & 8 & 200 & 20 & 33 & 57 & 113 & 446 & 657 & $6.336,60$ & $2.695,47$ & 729,9 \\
\hline & 833 & 22,5 & 4 & 500 & 12 & 300 & 20 & 29 & 63 & 165 & 318 & 590 & $3.993,12$ & $1.223,65$ & $-165,2$ \\
\hline & 1.111 & 28,5 & 4 & 550 & 8 & 250 & 20 & 49 & 72 & 142 & 576 & 848 & $10.579,93$ & $5.142,30$ & $2.190,73$ \\
\hline \multirow[t]{3}{*}{$60 \%$} & 833 & 25,5 & 4 & 600 & 10 & 300 & 20 & 40 & 83 & 177 & 407 & 716 & $7.976,16$ & $3.602,43$ & $1.244,01$ \\
\hline & 833 & 22,5 & 4 & 500 & 12 & 300 & 20 & 29 & 63 & 165 & 318 & 590 & $5.487,87$ & $2.136,96$ & 350,59 \\
\hline & 1.111 & 28,5 & 4 & 550 & 8 & 250 & 20 & 49 & 72 & 142 & 576 & 848 & $12.331,30$ & $6.233,17$ & 2.914, \\
\hline \multirow{3}{*}{$80 \%$} & 833 & 25,5 & 4 & 600 & 10 & 300 & 20 & 40 & 83 & 177 & 407 & 716 & 9.69 & $4.659,86$ & 1.936, \\
\hline & 833 & 22,5 & 4 & 500 & 12 & 300 & 20 & 29 & 63 & 165 & 318 & 590 & $6.982,62$ & $3.050,27$ & 943, \\
\hline & 1.111 & 28,5 & 4 & 55 & 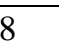 & 250 & 20 & 49 & 72 & 142 & 576 & 848 & 14.08 & $7.324,05$ & $3.639,2$ \\
\hline \multirow[t]{2}{*}{$100 \%$} & 833 & 25,5 & 4 & 600 & 10 & 300 & 20 & 40 & 83 & 177 & 407 & 716 & 11.41 & $5.717,30$ & 2.628 \\
\hline & 833 & 22,5 & 4 & 500 & 12 & 300 & 20 & 29 & 63 & 165 & 318 & 590 & $8.477,38$ & $3.963,58$ & $1.536,10$ \\
\hline
\end{tabular}

Em que: APV = Acréscimos percentuais no preço de venda da madeira desramada em relação a madeira nãodesramada; DI = Densidade Inicial de plantio (árvores/ha); $\mathrm{S}=$ Índice de sítio $(\mathrm{m}) ; \mathrm{I}_{1}=$ Idade do desbaste précomercial (anos); $\mathrm{NR}=$ Número de árvores remanescentes; $\mathrm{I}_{2}=$ Idade do $1^{\circ}$ desbaste comercial; $\mathrm{CF}=$ Idade do Corte Final; $\mathrm{VC1}=$ Volume comercial com diâmetro entre 8 e 17,9 cm; VC2 = Volume comercial com diâmetro entre 18 e $24,9 \mathrm{~cm}$; VC3 = Volume comercial com diâmetro entre 25 e 34,9 cm; VC4 = Volume comercial com diâmetro maior ou igual a $35 \mathrm{~cm} ; \mathrm{VT}=$ Volume Total.

\section{Efeito das taxas de desconto na definição dos regimes de manejo economicamente ótimos}

É destacada a sensibilidade dos lucros obtidos nos regimes de manejo testados em razão das variações nas taxas de desconto, ou seja, aumentos na taxa de desconto aumentam o risco do empreendimento, diminuindo a rentabilidade dos regimes de manejo simulados, conforme pode ser observado na Tabela 5 para as taxas de desconto de 6, 8 e 10\% a.a.

No sítio III, para a taxa de desconto de $8 \%$ a.a. a atividade de manejo com desbaste e desrama só será rentável, quando o acréscimo de preço da madeira desramada for igual ou superior a $20 \%$ do preço da madeira não-desramada. Para a taxa de desconto de $10 \%$ a.a. esta atividade só será rentável quando o acréscimo de preço da madeira desramada em relação a não-desramada for igual ou superior a $60 \%$. No caso do sítio II basta um acréscimo de preço igual ou superior a $20 \%$ para 
que a atividade seja rentável nessa mesma taxa de desconto.

Tomando como base um aumento de $60 \%$ no preço da madeira desramada em relação ao preço da madeira não-desramada, quando a taxa de desconto sobe de 6 para $8 \%$ a.a., o VPG dos sítios I, II e III cai 51,39, 54,83 e 61,06\% respectivamente. Se a taxa de desconto subir de 6 para $10 \%$ a.a. a queda do VPG desses sítios é de 79,29, 84,40 e 93,61\% a.a.

\section{Geração dos regimes de manejo para o cenário 2}

Na Tabela 8, são apresentados os regimes de manejo mais rentáveis, economicamente, para o cenário 2 para as taxas de desconto de 6,8 e $10 \%$ a.a. e para diferentes opções de preço de venda da madeira desramada em relação a madeira não-desramada. Verifica-se para uma taxa de desconto de $6 \%$ a.a., que o lucro obtido no sítio I varia de $\mathrm{R} \$ 6.659,05$ a $\mathrm{R} \$ 17.533,04$ por hectare. No sítio II, esse lucro é de $\mathrm{R} \$ 4.110,58$ a $\mathrm{R} \$ 13.350,33$ por hectare. Para o sítio III esses lucros estão entre R\$ $1.294,08$ a $\mathrm{R} \$ 8.930,08$ por hectare.

TABELA 8: Regimes de manejo, economicamente, mais rentáveis para o Cenário 2 para taxas de desconto de 6, 8 e $10 \%$ a.a. e para diferentes opções de preço de venda da madeira desramada.

\begin{tabular}{|c|c|c|c|c|c|c|c|c|c|c|c|c|c|c|c|c|c|}
\hline \multirow[t]{2}{*}{$\overline{\mathrm{APV}}$} & \multirow[t]{2}{*}{ DI } & \multirow[t]{2}{*}{$\bar{S}$} & \multirow[t]{2}{*}{$\mathrm{I}_{1}$} & \multirow[t]{2}{*}{ NR } & \multirow[t]{2}{*}{$\mathrm{I}_{2}$} & \multirow[t]{2}{*}{ NR } & \multirow[t]{2}{*}{\begin{tabular}{|l|l}
$\mathrm{I}_{3}$ & $\mathrm{I}$ \\
\end{tabular}} & \multirow[t]{2}{*}{ NR } & \multirow[t]{2}{*}{$\mathrm{CF}$} & \multirow[t]{2}{*}{$\mathrm{VC} 1$} & \multirow[t]{2}{*}{$\mathrm{VC} 2$} & \multirow[t]{2}{*}{ VC3 } & \multirow[t]{2}{*}{$\mathrm{VC} 4$} & \multirow[t]{2}{*}{$\mathrm{VT}$} & \multicolumn{3}{|c|}{ VPG } \\
\hline & & & & & & & & & & & & & & & $6 \%$ & $8 \%$ & $10 \%$ \\
\hline \multirow{4}{*}{$0 \%$} & 833 & 28,5 & 4 & 500 & 12 & 400 & 14 & 100 & 21 & 40 & 64 & 189 & 578 & 892 & $6.659,05$ & $2.773,52$ & 663,19 \\
\hline & 833 & 25,5 & 4 & 500 & 12 & 400 & 14 & 100 & 21 & 34 & 62 & 181 & 428 & 716 & $4.110,58$ & $1.322,28$ & $-180,7$ \\
\hline & 833 & 22,5 & 4 & 500 & 12 & 300 & 15 & 100 & 21 & 28 & 60 & 153 & 289 & 54 & $1.294,08$ & $-326,67$ & $-1.177,36$ \\
\hline & 833 & 28,5 & 4 & 500 & 12 & 400 & 14 & 100 & 21 & 40 & 64 & 189 & 578 & 89 & $8.883,85$ & $4.155,42$ & $1.597,99$ \\
\hline \multirow[t]{3}{*}{$20 \%$} & 833 & 25,5 & 4 & 500 & 12 & 400 & 14 & 100 & 21 & 34 & 62 & 181 & 428 & 716 & $5.937,87$ & $2.483,97$ & 633,84 \\
\hline & 833 & 22,5 & 4 & 500 & 12 & 400 & 15 & 100 & 21 & 28 & 61 & 166 & 288 & 560 & $2.777,08$ & 592,22 & $-573,34$ \\
\hline & 833 & 28,5 & 4 & 500 & 12 & 400 & 14 & 100 & 21 & 40 & 64 & 189 & 578 & 892 & $11.008,65$ & $5.547,73$ & $2.582,24$ \\
\hline \multirow{3}{*}{$40 \%$} & 833 & 25,5 & 4 & 500 & 12 & 400 & 14 & 100 & 21 & 34 & 62 & 181 & 428 & 716 & $7.765,16$ & $3.674,16$ & $1.439,63$ \\
\hline & 833 & 22,5 & 4 & 500 & 12 & 400 & 15 & 100 & 20 & 28 & 61 & 167 & 265 & 539 & 4.28 & $1.574,58$ & 105,20 \\
\hline & 833 & 28,5 & 4 & 500 & 12 & 400 & 14 & 100 & 21 & 40 & 64 & 189 & 578 & 892 & 13.18 & 950,57 & $3.533,76$ \\
\hline \multirow[t]{3}{*}{$60 \%$} & 833 & 25,5 & 4 & 500 & 12 & 400 & 14 & 100 & 20 & 34 & 62 & 182 & 394 & 68 & 9.66 & 4.8 & $2.366,94$ \\
\hline & 833 & 22,5 & 4 & 500 & 12 & 400 & 15 & 100 & 20 & 28 & 61 & 167 & 265 & 539 & $5.883,55$ & $2.550,18$ & 758,99 \\
\hline & 833 & 28,5 & 4 & 500 & 12 & 400 & 14 & 100 & 21 & 40 & 64 & 189 & 578 & 892 & $15.358,24$ & $8.353,41$ & $4.485,28$ \\
\hline \multirow[t]{3}{*}{$80 \%$} & 833 & 25,5 & 4 & 500 & 12 & 400 & 14 & 100 & 20 & 34 & 62 & 182 & 394 & 684 & $11.479,40$ & $6.054,53$ & $3.051,21$ \\
\hline & 833 & 22,5 & 4 & 500 & 12 & 400 & 15 & 100 & 20 & 28 & 61 & 167 & 265 & 539 & $7.381,81$ & $3.525,79$ & $1.412,77$ \\
\hline & 833 & 28,5 & 4 & 500 & 12 & 400 & 14 & 100 & 21 & 40 & 64 & 189 & 578 & 892 & $17.533,04$ & $9.756,25$ & $5.436,79$ \\
\hline \multirow[t]{2}{*}{$100 \%$} & 833 & 25,5 & 4 & 500 & 12 & 400 & 14 & 100 & 20 & 34 & 62 & 182 & 394 & 684 & $13.350,33$ & $7.244,72$ & $3.857,01$ \\
\hline & 833 & 22,5 & 4 & 500 & 12 & 400 & 15 & 100 & 20 & 28 & 61 & 167 & 265 & 539 & $8.930,08$ & $4.501,40$ & $2.066,56$ \\
\hline
\end{tabular}

Em que: $\mathrm{APV}=$ Acréscimos percentuais no preço de venda da madeira desramada em relação a madeira nãodesramada; DI = Densidade Inicial de plantio (árvores/ha); $\mathrm{S}=$ Índice de sítio $(\mathrm{m}) ; \mathrm{I}_{1}=$ Idade do desbaste précomercial (anos); $\mathrm{NR}=$ Número de árvores remanescentes; $\mathrm{I}_{2}=$ Idade do $1^{\circ}$ desbaste comercial; $\mathrm{I}_{3}=$ Idade do $2^{\circ}$ desbaste comercial; $\mathrm{CF}=$ Idade do Corte Final; $\mathrm{VC1}=$ Volume comercial com diâmetro entre 8 e 17,9 cm; VC2 = Volume comercial com diâmetro entre 18 e 24,9 cm; VC3 = Volume comercial com diâmetro entre 25 e 34,9 cm; VC4 $=$ Volume comercial com diâmetro maior ou igual a $35 \mathrm{~cm}$; VT $=$ Volume Total.

Observa-se também que, quando o preço da madeira desramada é o dobro do preço da madeira não-desramada, o acréscimo no VPG é de 163,3\% para o sítio I, de 224,8\% para o sítio II e 
de $590,1 \%$ para o sítio III.

A densidade inicial de plantio influencia a produtividade e, conseqüentemente, a rentabilidade dos regimes de manejo simulados. O plantio de 833 árvores/ha, espaçamento $3 \mathrm{x} 4 \mathrm{~m}$, foi o que proporcionou a maior rentabilidade para as três classes de sítio consideradas. Esse fato já era esperado para as classes de sítio I e II, já que, como foi mencionado anteriormente, as árvores em sítios mais produtivos apresentam acelerado ritmo de crescimento em diâmetro após a realização de cada desbaste. No entanto, contrariamente ao esperado para a classe de sítio III, o espaçamento que mais se destacou também foi o $3 \times 4 \mathrm{~m}$. Esse fato, provavelmente, ocorreu em face dos custos de realizar 3 desbastes em um número maior de indivíduos terem sido proporcionalmente superiores ao volume gerado para fins mais nobres de uso da madeira.

\section{Efeito das taxas de desconto na definição dos regimes de manejo economicamente ótimos}

É destacada a sensibilidade dos lucros obtidos nos regimes de manejo testados em razão das variações nas taxas de desconto. No sítio III, para a taxa de desconto de $8 \%$ a.a. a atividade de manejo com desbaste e desrama só será rentável quando o acréscimo no preço da madeira desramada for igual ou superior a $20 \%$ do preço da madeira não-desramada. Para a taxa de desconto de $10 \%$ a.a. esta prática de manejo só será viável para o sítio III, quando o preço da madeira desramada for igual ou superior a $40 \%$ do preço da madeira não-desramada. No caso do sítio II, para a taxa de $8 \%$ a.a., a desrama é rentável mesmo que não haja acréscimo no preço da madeira desramada, mas se a taxa for de $10 \%$ a.a., é necessário que o preço suba $20 \%$ para que a desrama seja viável economicamente.

Tomando como base um aumento de $60 \%$ no preço da madeira desramada em relação ao preço da não-desramada, quando a taxa de desconto sobe de 6 para $8 \%$ a.a., o VPG dos sítios I, II e III cai $47,28,49,37$ e $56,65 \%$, respectivamente. Se a taxa passar de 6 para $10 \%$ a.a. a queda do VPG desses sítios é de 73,20, 75,37 e 87,10\% a.a.

\section{Produção volumétrica dos regimes de manejo economicamente mais rentáveis para os dois cenários em estudo}

Observa-se, ao comparar as Tabelas 7 e 8 que, para os sítios I e II, os regimes de manejo, sujeitos a 1 desbaste pré-comercial e 2 desbastes comerciais (cenário 2), são economicamente mais rentáveis que os regimes de manejo sujeitos a 1 desbaste pré-comercial e 1 desbaste comercial (cenário 1), considerando todas as taxas de desconto e os diferentes preços de venda da madeira desramada. Nota-se que a adoção de 3 desbastes eleva a produção volumétrica nas maiores classes de diâmetro nesses sítios, elevando-se assim os VPGs desses regimes de manejo. Essa superioridade é de tal intensidade que se o preço da madeira desramada for $60 \%$ superior ao da madeira não-desramada, os acréscimos de VPG, para os sítios I, II e III são respectivamente, 24,6\%, 20,5\% e $7,2 \%$. A tendência é de redução dessa diferença quanto maior o diferencial de preço da madeira desramada para a não-desramada e quanto menos produtivo for o sítio.

Para o sítio III, considerando o preço da madeira desramada igual ao preço da madeira não-desramada, é a adoção de 2 desbastes que produz um maior volume na maiores classes de diâmetro, 
refletindo num maior VPG. No entanto, ao considerarmos acréscimos de 20 a $100 \%$ no preço da madeira desramada, nota-se que a adoção de 3 desbastes passa a ser mais atrativa do ponto de vista econômico, apesar do menor volume produzido nas maiores classes de diâmetro.

\section{Comparação entre os cenários 1 e 2 com base em taxas de desconto de 8 e $10 \%$ a.a.}

Para as taxas de desconto de 8 e $10 \%$ a.a. o cenário 2 (Tabela 8), com 1 desbaste pré- -comercial e 2 desbastes comerciais novamente é superior ao cenário 1 (Tabela 7) com 1 desbaste pré-comercial e outro comercial, exceto para o sítio III considerando o preço da madeira desramada igual ao preço da madeira não-desramada. Essa superioridade é tal que, para a taxa de desconto de $8 \%$ a.a., se o preço da madeira desramada for $60 \%$ superior ao da madeira nãodesramada, os acréscimos de VPG, para os sítios I, II e III são respectivamente 32,1, 33,5 e 18,7\%.

\section{CONCLUSÕES}

Preferencialmente, o manejo conjugado desbaste/desrama deve ser implementado em sítios mais produtivos. No caso, esse estudo indica os índices de sítio 28,5 e 25,5 $\mathrm{m}$ na idade de referência de 20 anos, para Pinus taeda.

A condução de regimes de manejo com a realização de um desbaste pré-comercial, seguido de 2 desbastes comerciais e desrama, deve ser adotada, por ser essa a opção que gera maiores receitas líquidas para os sítios I e II. Para o sítio III, a melhor opção é a adoção de regimes de manejo com um desbaste pré-comercial seguido de 1 desbaste comercial e desrama.

A densidade inicial de plantio ideal, quando 1 desbaste pré-comercial seguido de desrama e de 1 desbaste comercial são adotados para os sítios I e II é de 833 árvores/ha, enquanto que para o sítio III, é de 1.111 árvores/ha.

A densidade inicial de plantio ideal quando 1 desbaste pré-comercial seguido de desrama e de 2 desbastes comerciais são adotados para os sítios I, II e III, é de 833 árvores/ha.

Para taxas de desconto de $10 \%$ a.a., no sítio I, há realização de lucro, considerando qualquer situação de preço de venda da madeira desramada. No sítio II, somente quando o preço de venda da madeira podada for $20 \%$ superior é que haverá lucro. Para o sítio III, somente quando o preço de venda da madeira desramada for $60 \%$ superior ao preço da madeira não-desramada é que haverá lucro.

Para taxas de desconto de $8 \%$ a.a., nos sítios I e II, há realização de lucro, considerando qualquer situação de preço de venda da madeira desramada. Para o sítio III, somente quando o preço de venda da madeira desramada for $20 \%$ superior ao preço da madeira não-desramada é que haverá lucro.

Para taxas de desconto de $6 \%$ a.a., considerando qualquer situação de preço de venda da madeira desramada, os plantios nos sítios I, II e III dão lucro quando sujeitos ao manejo conjugado desbaste/desrama. 


\section{REFERÊNCIAS BIBLIOGRÁFICAS}

ACERBI JUNIOR, F.W. Definição de regimes de desbastes e poda economicamente ótimos para Pinus taeda . 1998, 177p. Dissertação (Mestrado em Engenharia Florestal) - Universidade Federal de Lavras, Lavras.

BAILEY, R.L.; WARE, K.D. Compatible basal area growth and yield model for thinning and unthinned stand. Canadian Journal of Forest Research, Otawa, v. 13, p. 563 - 571, 1983.

BENTLEY, W.; TEENGUARDEN, D. Financial maturity: a theory review. Forest Science, v. 11, n. 3, p. 76 $-87,1965$.

BERTOLOTI, G.; SIMÕES, J. W.; NICOLIELO, N. et al.. Efeitos de diferentes métodos e intensidades de desbaste na produtividade de Pinus caribaea var hondurensis Barr. et Golf. IPEF, Piracicaba, n.24, p.4753, ago.1983.

BURKHART, H.E. Data collection and modeling approaches for forest crowth and yield prediction. In: Predicting Forest Crowth and Yield: Current issues, future prospects. Seatle: University Washington, 1987. p. $3-16$.

BURKHART, H.E.; CAO, Q.V.; WARE, K.D. A comparation of growth and yield prediction models for loblolly pine. Virginia: School of Forestry and Wildlife Resources, 1981. 59 p. (Publ. FWS, 2).

FINGER, C.A.G.; SCHNEIDER, P.R. Determinação do peso do desbaste para florestas de Eucalyptus grandis Hill ex Maiden, com base no índice de espaçamento relativo. Ciência Florestal, v.9, n.1, p. 7987, 1999.

GOMES, F.S. A seleção de regimes de manejo mais rentáveis em Pinus taeda L. na produção de madeira para papel e celulose. 1999. 137p. Dissertação (Mestrado em Ciências Florestais) - Setor de Ciências Agrárias, Universidade Federal do Paraná, Curitiba.

HAFLEY, W.L.; BUFORD, M.A. A bivariate model for growth and yield prediction. Forest Science, Peking, v. 31, n. 1, p. 237 - 247, Mar. 1985.

KNOEBELL, B.C.; BURKHART, H. E.; BECK, D. E. A growth and yield model for thinned stands of yellow - poplar. Forest Science, Bethesda, v. 32, n. 2, p. 1 - 62, June 1986. (Monograph, 27)

MACEDO, A.R.P.; MATTOS, R.L.G. Produtos florestais madeira em tora, madeira serrada e painéis: Panorama 1980/1992. S.1.: BNDES/FINAME/BNDESPAR, 1995. 41p.

MAGIN, E.K. Standortgerechte ertragsmittlung als teil der forsteinrichtung. Mitteilungen aus der staatsforst - erwaltung Bayerns, v. 34, p. 305 - 314. 1964.

OLIVEIRA, E. B. Um sistema computadorizado de prognose do crescimento e produção de Pinus taeda L., com critérios quantitativos para avaliação técnica e econômica de regimes de manejo. 1995. 134p. Tese (Doutorado em Ciências Florestais) - Setor de Ciências Agrárias, Universidade Federal do Paraná, Curitiba.

SCHNEIDER, P.R. Betriebswirtschaftliche und ertragskundliche Grundlagen der Forsteinrichtung in Sudbrasilien am Beispiel von Pinus elliottii. 1984. 190p. Tese (Doutorado) - Diss. da Albert - Ludwigs - Universitat. Freiburg, Freiburg, Alemanha. Efeito da intensidade do desbaste sobre o fator K - Magin em povoamentos de Pinus elliottii E. In: 
CONGRESSO FLORESTAL PANAMERICANO, 1.; CONGRESSO FLORESTAL BRASILEIRO, 7., 1993, Curitiba. Anais... Curitiba: SBS - SBEF, 1993. p. $504-507$.

SCOLFORO, J.R.S; ACERBI JUNIOR, F.W.; MELLO, J.M. et al.. A model for evaluation of forest growth and yield with thinning simulator. Forest Ecology and Management. 1999. 20p. (submetido)

SCOLFORO, J.R.S. Sistema integrado para predição e análise presente e futura do crescimento e produção, com otimização de remuneração de capitais, para Pinus caribaea var. hondurensis. 1990. 290p. Tese (Doutorado em Ciências Florestal) - Setor de Ciências Agrárias, Universidade Federal do Paraná, Curitiba.

. O sistema PISAPRO. Lavras: UFLA/FAEPE, 1997. 79p.

SCOLFORO, J.R.S.; MACHADO, S.A. Um sistema de crescimento e produção com simulador de desbaste. Scientia Forestalis, Piracicaba, n.50, p.51-64, dez.1996.

SCOLFORO, J.R.S.; MAESTRI, R. O manejo de florestas plantadas. In: SCOLFORO, J.R.S. Manejo florestal. Lavras: UFLA/FAEPE, 1997. Cap. 8. p. 313-379.

SCOLFORO, J.R.S. Modelagem do crescimento e da produção de florestas plantadas e nativas. Lavras: UFLA/FAEPE, 1998a. 441p.

_. O sistema INVEST. Lavras: UFLA/FAEPE, 1998b. 52p. 